\title{
Self-assembled "nanocubicle" as a carrier for peroral insulin delivery
}

\author{
H. Chung, J. Kim, J. Y. Um, I. C. Kwon, S. Y.Jeong \\ Biomedical Research Center, Korea Institute of Science and Technology, Seoul, Korea
}

\begin{abstract}
Aims/hypothesis. A patient with (insulin-dependent) diabetes mellitus receives at least one subcutaneous insulin injection a day to maintain low serum glucose concentrations. Since patients' compliance with such dosage regimens is too low, the development of an oral formula is clearly attractive. We present the development of a liquid formula that can be easily dispersed in water to produce particles named "nanocubicles" which efficiently encapsulate insulin.

Methods. Fasted streptozotocin-induced diabetic rats were administered orally with particles encapsulating insulin, and particles without insulin or soluble insulin in water. Groups of rats were also injected soluble insulin in PBS for control. Blood glucose concentration and insulin concentration were measured 1,2,3,
\end{abstract}

4 and $6 \mathrm{~h}$ after the administration of the insulin formulas.

Results. In vitro experiments show that the particles can be taken up by the Caco-2 cells at a high ratio. The serum glucose concentration was controlled for more than $6 \mathrm{~h}$ after oral insulin administration but returned to the basal concentration in $3 \mathrm{~h}$ when $1 \mathrm{IU} / \mathrm{kg}$ of insulin was injected intravenously.

Conclusion/interpretation. Our biocompatible and stable oral insulin formulation is easy to prepare and produces reproducible hypoglycaemic effects, therefore we anticipate clinical acceptance and utilization of this form of insulin therapy. [Diabetologia (2002) 45: 448-451]

Keywords Peroral, oral, insulin, delivery, self-assembled, nanocubicles, liquid formulation, diabetes.
Many scientists have endeavoured to search for oral insulin formula by using polymer or lipid systems [1-6], protease inhibitors and permeation enhancers [7-10]. Other routes have also been explored as alternatives to insulin injections [11-13]. Although these formulations achieved varying degrees of success, there are many obstacles that must be overcome if clinically successful formulas are to be achieved.

We developed a homogeneous liquid formula that has distinctly different physical properties from those of the pre-existing formulas. By simply mixing

Received: 10 September 2001 and in revised form: 9 November 2001

Corresponding author: Dr. S. Y. Jeong, Biomedical Research Center, Korea Institute of Science and Technology, 39-1 Hawolkok-dong, Sungbuk-ku, Seoul 136-791, Korea, e-mail: syjeong @kist.re.kr our liquid formula in excess water or phosphate buffered saline (PBS, $\mathrm{pH}$ 7.4), nano-sized particles ranging from 200 to $500 \mathrm{~nm}$ are formed. Encapsulation efficiency of insulin inside the particles was 70 to $100 \%$. The formula can be made without heat or physical force and can be sterilized easily by filtration.

We name the submicron-sized lipid particles formed spontaneously in water 'nanocubicles'. Nanocubicles can be similar to, but differ in some respects from, the so-called cubosomes [14, 15]. For preparation of nano-sized cubosomes, the mixture of monoolein, emulsifier and water needs to be microfluidized at about $80^{\circ} \mathrm{C}$ and cooled slowly to room temperature. Since insulin cannot be stable under this harsh condition, cubosomes have been used in the form of large aggregates $(10 \mu \mathrm{m} \sim 1 \mathrm{~mm})$, prepared at room temperature by stirring, to test the possibility of their being used as a nasal insulin delivery system [16] but not as an oral formula [17]. 
A
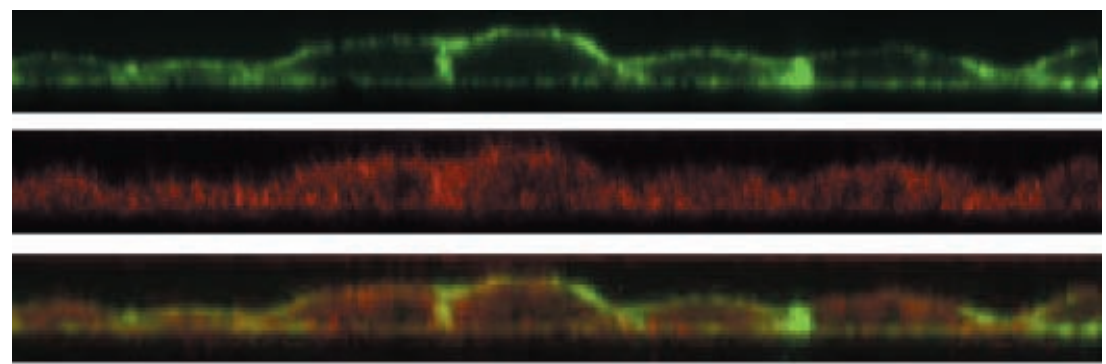

B

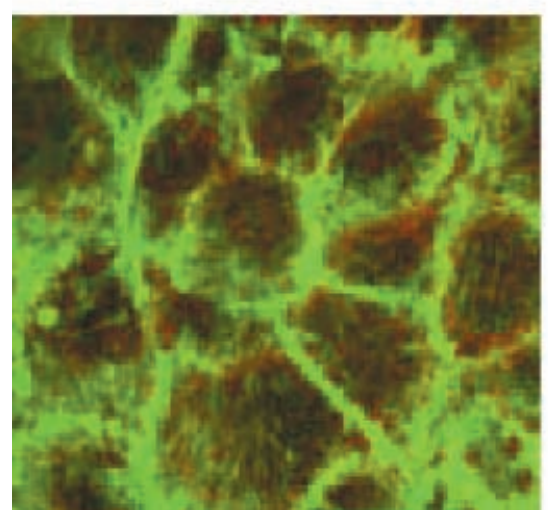

Fig. 1 (A, B). Confocal laser scanning microscopy of the rhodamine-labelled lipid nanocubicles. The particles were applied to Caco-2 cells for $3 \mathrm{~h}$. A Vertical section showing green fluorescence of cytoskeleton (top), red rhodamine fluorescence (middle), and their superposition (bottom) show that nanocubicles were taken up by the cells. B Horizontal section of the cells incubated with Rhodamine-PE labelled nanocubicles (left) and the negative control (right)

\section{Materials and methods}

Preparation of liquid formulation. One hundred milligrams of monoolein were mixed homogeneously with $20 \mathrm{mg}$ of Pluronic F-127, $280^{\circ} \mu \mathrm{l}$ of ethanol and $280^{\circ} \mu \mathrm{l}$ of propylene glycol. After adding $8^{\circ} \mu \mathrm{l}$ of $1 \mathrm{~N}$ hydrochloric acid into the mixture, $8 \mathrm{mg}$ of insulin powder was added and stirred until it was clear. Then ethanol in the mixture was evaporated completely in the mixture under a vacuum to prepare the liquid formula containing insulin. To prepare the liquid formulation containing 1, 2-dioleyl-sn-glycero-3-phosphoethanolamine-N-(lissamine rhodamine B sulfonyl) (Rhodamine-PE), the mixture of $5 \mathrm{mg}$ of Rhodamine PE and $100 \mathrm{mg}$ of monoolein was used instead of $100 \mathrm{mg}$ of monoolein.

Formation of nanocubicles and size determination. Nanocubicles are formed by vortexing gently after adding $100^{\circ} \mu \mathrm{l}$ of liquid formula in $500^{\circ} \mu 1$ to $1 \mathrm{ml}$ of water or PBS. The average particle size of nanocubicles was determined by quasielastic laser light scattering with a Malvern Zetasizer (Malvern Instruments, Worcester, England).

Encapsulation efficiency of insulin in nanocubicles. The dispersion containing nanocubicles was added into the reservoir of

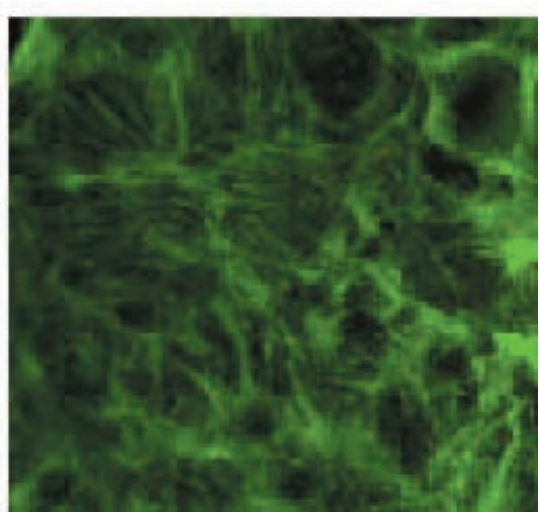

Centricon (YM-100, Amicon, Millipore, Bedford, Mass., USA). The filtrate, that contains free Rhodamine-PE or insulin, was collected after centrifuging the dispersion to determine the encapsulation efficiency.

Caco-2 cell culture. Caco- 2 cells were cultured at $37^{\circ} \mathrm{C}, 5 \%$ $\mathrm{CO}_{2}$ in DMEM supplemented with $10 \%$ fetal bovine serum, $1 \%$ non-essential amino acids and $1 \%$ penicillin-streptomycin. The cells were plated at a density of 5000 cells $/ 1.8 \mathrm{~cm}^{2}$ on Lab-Tek 4-well chamber slides and cultured for 2 days before use.

In vitro particle uptake test. Cell culture media were removed and the cells were washed three times with cold Hanks' balanced salt solution. After adding $1 \mathrm{ml}$ of the dispersion corresponding to $0.5 \mathrm{mg}$ of rhodamine-PE to each chamber, the cells were incubated for $3 \mathrm{~h}$ at $37^{\circ} \mathrm{C}$. Cells were washed and incubated with $4 \%$ paraformaldehyde in PBS for $30 \mathrm{~min}$ and were stained with phalloidin-FITC. The slides were mounted on fluorescent mounting medium (DAKO) and analysed with a confocal laser scanning microscope (MRC-1024, Bio-Rad, Hercules, Calif., USA) with $\mathrm{Ar} / \mathrm{Kr}$ ion laser.

Animals. We followed the National Institutes of Health (NIH) guidelines for the care and use of laboratory animals (NIH publication 85-23 Rev. 1985). Male Wistar rats, 5 weeks old, weighing 120 to $150 \mathrm{~g}$ (Charles River Japan, Yokohama, Japan) were housed in groups of four in separate cages with free access to food and water for 1 week in a temperature-regulated and humidity-regulated room under a 12-h light/dark cycle prior to the experiments. Diabetes was induced by three consecutive intraperitoneal injections of streptozotocin $(45 \mathrm{mg} / \mathrm{kg})$. After 2 weeks, rats were considered diabetic when the blood glucose concentrations was above $16.7 \mathrm{mmol} / \mathrm{l}$ at the fasting state. 


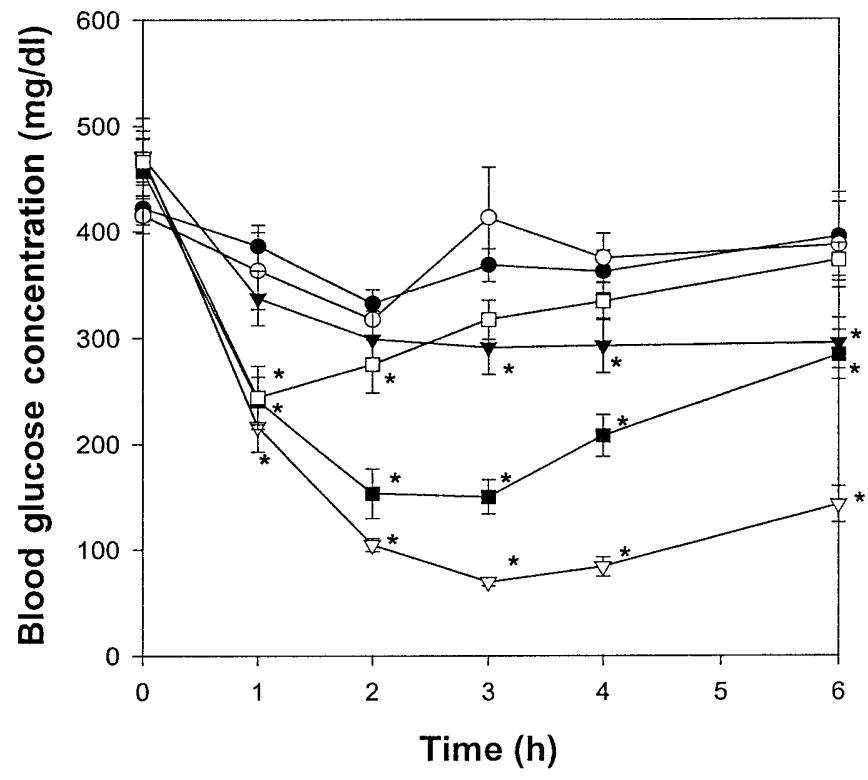

Fig. 2. Blood glucose concentrations in diabetic rats after oral administration of the nanocubicles without insulin $(\bullet, n=6)$, insulin solution $(\bigcirc, 50 \mathrm{IU} / \mathrm{kg}$ body weight, $n=6)$, nanocubicles with insulin $(\boldsymbol{\nabla}, 30 \mathrm{IU} / \mathrm{kg}$ body weight, $n=22 ; \nabla, 50 \mathrm{IU} / \mathrm{kg}$ body weight, $n=18$ ). Insulin solutions were also injected through the tail vein as controls $(\boldsymbol{\square}, 5 \mathrm{IU} / \mathrm{kg}$ body weight, $n=6 ; \square, 1 \mathrm{IU} / \mathrm{kg}$ body weight, $n=3)$. Data are represented as means \pm SEM. $* p<0.05$ compared to insulin solution oral feeding (one-way ANOVA followed by Scheffe's F test for post hoc pairwise comparisons)

Biological activity of insulin. Insulin-encapsulated nanocubicles were tested for biological activity by intravenous injection of the dispersion (5 IU/ $\mathrm{kg}$ as insulin) into fasted rats. Only biologically active formulas were used for oral feeding.

Oral administration of nanocubicles encapsulating insulin. The baseline blood glucose concentration was measured in rats fasted for $4 \mathrm{~h}$. During the experiment the rats were kept at a fasting state but with free access to water. After the oral or intravenous administration of the insulin formulas, blood samples were drawn from the tail vein using a 26 gauge needle under light anesthesia to measure glucose and insulin concentrations.

Determination of the blood glucose and insulin concentration. The whole blood glucose concentration was measured immediately after the blood collection using an Accutrend Alpha (Roche Diagnostics, Cham, Switzerland). The serum insulin concentration was determined using a radioimmunoassay kit (COAT-A-COUNT Insulin, Diagnostic Product, Los Angeles, Calif., USA).

\section{Results}

Characteristics of nanocubicles. The average size and polydispersity of the nanocubicles containing Rhodamine-PE were $248 \mathrm{~nm}$ and 0.344 , respectively, and those containing insulin were $220 \mathrm{~nm}$ and 0.235 , respectively. The encapsulation efficiency of Rhoda-
mine-PE and insulin were $97 \%$ and $100 \%$, respectively.

In vitro uptake of nanocubicles into Caco- 2 cells. The nanocubicles containing Rhodamine-PE was used for in vitro experiments. After $3 \mathrm{~h}$ of exposure of nanocubicles to Caco- 2 cells, the particles were found within the cytoplasm indicating a rapid uptake mechanism (Fig.1). Vertical (Fig.1A) and horizontal (Fig.1B, left) sections of Caco-2 cells show that red rhodamine fluorescence seems to co-locate with the green fluorescence of cytoskeleton stained by phalloidin-FITC. In comparison, soluble rhodamine, Rhodamine-PE or the particles without RhodaminePE did not show any red intracellular fluorescence (Fig. 1B, right). When the lipid emulsion made of soybean oil, egg phosphatidylcholine and Rhodamine$\mathrm{PE}$ was used, the intracellular fluorescence was much weaker $(20 \%)$ than that with our nanocubicle system (data not shown).

Uptake of nanocubicles via oral administration. Our next step was to carry out in vivo experiments to evaluate the hypoglycaemic effect of the insulin-loaded nanocubicles (Fig. 2). Three groups of fasted streptozotocin-induced diabetic rats were administered orally with particles encapsulating insulin (50 or 30 $\mathrm{IU} / \mathrm{kg}$ ), particles without insulin or soluble insulin in water $(50 \mathrm{IU} / \mathrm{kg})$. Groups of rats were injected $5 \mathrm{IU} /$ $\mathrm{kg}$ or $1 \mathrm{IU} / \mathrm{kg}$ of soluble insulin in PBS for control. Both the empty particles and the insulin solution did not show any hypoglycaemic effect. As expected, the serum glucose concentration was lowered by the 1 $\mathrm{IU} / \mathrm{kg}$ insulin injection in $1 \mathrm{~h}$ but gradually recovered to the original concentration in $6 \mathrm{~h}$. A more interesting result was obtained when the particles encapsulating insulin $(50 \mathrm{IU} / \mathrm{kg})$ were given orally. The hypoglycaemic effect was noticed in $1 \mathrm{~h}$ and lasted for the duration of the experiment $(6 \mathrm{~h})$ and the glucose concentration was controlled at 1.7 to $6.7 \mathrm{mmol} / \mathrm{l}$ for the whole period compared to those of untreated rats (16.7-22.2 mmol/l). Of interest, the hypoglycaemic effect was very fast considering the mechanism of the absorption after oral feeding.

The serum insulin concentration was also analysed for the above groups of rats (Fig. 3). When empty nanocubicles or insulin solution was fed, the serum insulin concentration did not deviate from the basal concentration for $6 \mathrm{~h}$. With $1 \mathrm{IU} / \mathrm{kg}$ injection, the insulin concentration was slightly, but significantly higher $(p<0.05)$ than the basal concentration for $4 \mathrm{~h}$. The serum insulin concentration, however, increased rapidly to $140^{\circ} \mu \mathrm{U} / \mathrm{ml} 1 \mathrm{~h}$ after the $5 \mathrm{IU} / \mathrm{kg}$ insulin injection but declined slowly to the basal concentration. The insulin concentration for the groups of rats fed with the nanocubicles encapsulating insulin increased significantly $(p<0.05)$ from the normal value. The insulin concentration was at its maximum in 1 to about 


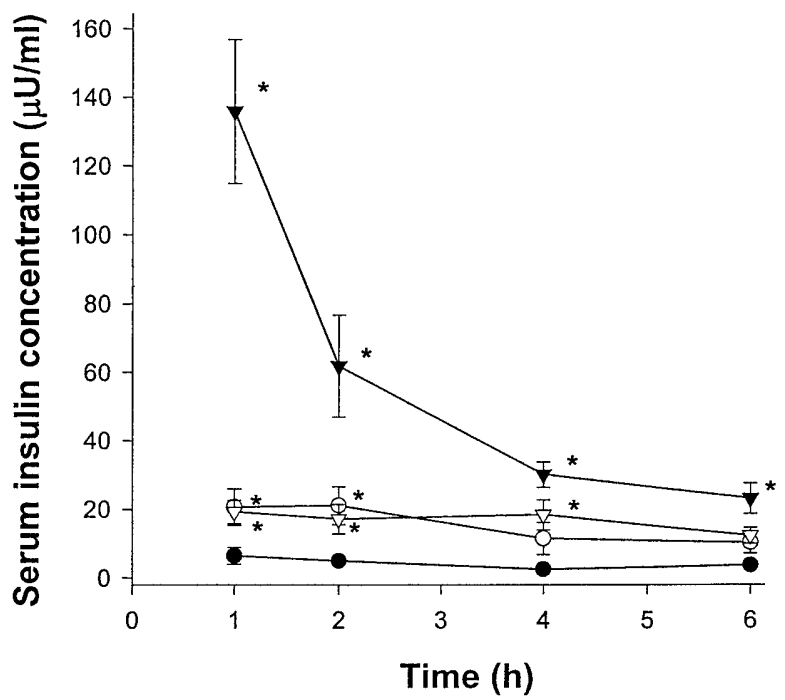

Fig. 3. Serum insulin concentrations in diabetic rats after oral administration of the nanocubicles without insulin $(\bullet, n=7)$, nanocubicles with insulin $(\bigcirc, 50 \mathrm{IU} / \mathrm{kg}$ body weight, $n=5)$. Insulin solutions were also injected through the tail vein as controls $(\boldsymbol{\nabla}, 5 \mathrm{IU} / \mathrm{kg}$ body weight, $n=6 ; \nabla, 1 \mathrm{IU} / \mathrm{kg}$ body weight, $n=3$ ). Data are represented as means \pm SEM. $* p<0.05$ compared to insulin solution oral feeding (one-way ANOVA followed by Scheffe's F test)

$2 \mathrm{~h}$, and though the insulin concentration declined in 4 to about $6 \mathrm{~h}$, it was still higher than at the baseline.

\section{Discussion}

The aim of our new precursor-type liquid formula of nanocubicles was to overcome some of the problems involving preparation of the cubosomes, while retaining many outstanding properties of an ideal oral peptide delivery system. In addition to the easy preparation procedure and stability upon storage, insulin does not contact, and is shielded from, proteolytic enzymes because it is encapsulated in the particles. We expect that nanocubicles will adsorb in the intestinal epithelia since monoolein, or the cubic phase that it forms in water is known to be mucoadhesive [18].

We do not know whether the particles themselves increase the intestinal penetration or merely protect insulin from the attack by the proteolytic enzymes. Our biocompatible formula is easy to prepare, to store and to administer into the body with a superior effect in controlling hyperglycaemia in a reproducible and predictable manner. These characteristics constitute some of the attractive points in our novel development of an oral insulin formulation.

Acknowledgements. This work was supported by the KIST2000 project.

\section{References}

1. Langer R, Folkman J (1995) Polymers for the sustained release of proteins and other macromolecules. Nature 263: 797-800

2. Mathiowitz E, Jacob JS, Jong YS et al. (1997) Biologically erodable microspheres as potential oral drug delivery systems. Nature 386: 410-414

3. Carino GP, Jacob JS, Mathiowitz E (2000) Nanosphere based oral insulin delivery. J Controlled Releau 65: 261-269

4. Iwanaga K, Ono S, Narioka K et al. (1997) Oral delivery of insulin by using surface coating liposomes improvement of stability of insulin in GI tract. Int J Pharm 157: 73-80

5. Mesiha M, Sidhom M (1995) Increased oral absorption enhancement of insulin by medium viscosity hydroxypropyl cellulose. Int J Pharm 114: 137-140

6. Takeuchi H, Yamamoto H, Niwa T, Hino T, Kawashima Y (1996) Enteral absorption of insulin in rats from mucoadhesive chitosan-coated liposomes. Pharmacol Res 13: 896-901

7. Carino GP, Mathiowitz E (1999) Oral insulin delivery. Adv Drug Deliv Rev 35: 245-257

8. Silva-Cunha A, Cheron M, Grossiord JL, Puisieux F, Seiller $\mathrm{M}$ (1998) W/O/W multiple emulsions of insulin containing a protease inhibitor and an absorption enhancer: biological activity after oral administration to normal and diabetic rats. Int J Pharm 169: 33-44

9. Morishita M, Morishita I, Takayama K, Machida Y, Nagai T (1993) Site-dependent effect of aprotinin, sodium caprate, $\mathrm{Na}_{2}$ EDTA and sodium glycocholate on intestinal absorption of insulin. Biol Pharm Bull 16: 68-72

10. Fix J (1996) Oral controlled release technology for peptides: status and future prospects. Pharm Res 13: 1760-1764

11. Patton JS, Bukar J, Nagarajan S (1999) Inhaled insulin. Adv Drug Deliv Rev 35: 235-247

12. Selam JL (1999) Implantable insulin pumps. Lancet 354: 178-179

13. Renard E et al. (1999) Improved stability of insulin delivery from implanted pumps using a new preparation process for infused insulin. Diabetes Care 22: 1371-1372

14. Gustafsson J, Ljusberg-Wahren H, Almgren M, Larsson K (1997) Submicron particles of reversed lipid phases in water stabilized by a nonionic amphiphjilic powder. Langmuir 13: 6964-6971

15. Larsson K (1999) Colloidal dispersion of ordered lipid-water phases. J Disp Sci Tech 20: 27-34

16. Landh T, Larsson K (1996) Particles, method of preparing said particles and uses thereof. United States patent No. 5531925

17. Desai MP, Labhasetwar V, Amidon GL, Levy RJ (1996) Gastrointestinal uptake of biodegradable microparticles: Effect of particle size. Pharm Res 13: 1838-1845

18. Nielsen LS, Schubert L, Hansen J (1998) Bioadhesive drug delivery systems I. Characterisation of mucoadhesive properties of systems based on glyceryl mono-oleate and glyceryl monolinoleate. Eur. J. Pharm. Sci. 6: 231-239 